\title{
Treatment of Hidradenitis Suppurativa Patient with Klinefelter Syndrome by Adalimumab
}

\author{
Ji Yeoun Shin, Jung Yeon Hong ${ }^{1}$, Ho Jung Lee ${ }^{1}$, Chang Yoon Sim ${ }^{1}$, Young Lip Park, Jong Suk Lee ${ }^{1}$, \\ Sung Yul Lee ${ }^{1}$, Jung Eun Kim ${ }^{1}$ \\ Department of Dermatology, Soon Chun Hyang University Bucheon Hospital, Bucheon, ${ }^{1}$ Department of Dermatology, Soon Chun Hyang \\ University Cheonan Hospital, Cheonan, Korea
}

\begin{abstract}
Hidradenitis suppurativa (HS) is a chronic, inflammatory and painful skin disease with recurrent nodules and tracts involving the intertriginous regions. It is known that the patient with HS shows an increased risk of metabolic disorders such as diabetes, metabolic syndrome and autoimmune diseases. Klinefelter syndrome (KS) is a sex chromosomal disorder occurring in males due to an abnormality of sexual differentiation, characterized by 47, XXY karyotype. Also, KS is related with somatic comorbidities such as metabolic syndrome, autoimmune and rheumatologic disorders as HS is. We report a HS patient with KS who shows a big improvement while on tumor necrosis factor-alpha inhibitor treatment. (Ann Dermatol 31(4) 446 449, 2019)
\end{abstract}

\section{-Keywords-}

Hidradenitis suppurativa, Klinefelter syndrome

\section{INTRODUCTION}

Hidradenitis suppurativa (HS) is a chronic recurrent suppurative skin disorders, mostly involving axillae, inguinal

Received March 9, 2018, Revised June 22, 2018, Accepted for publication July 6, 2018

Corresponding author: Jung Eun Kim, Department of Dermatology, Soon Chun Hyang University Cheonan Hospital, 31 Suncheonhyang 6-gil, Dongnam-gu, Cheonan 31151, Korea. Tel: 82-41-570-2270, Fax: 82-41-570-2271, E-mail: freesia0210@naver.com

ORCID: https://orcid.org/0000-0002-8399-8456

This is an Open Access article distributed under the terms of the Creative Commons Attribution Non-Commercial License (http://creativecommons. org/licenses/by-nc/4.0) which permits unrestricted non-commercial use, distribution, and reproduction in any medium, provided the original work is properly cited.

Copyright (c) The Korean Dermatological Association and The Korean Society for Investigative Dermatology area, buttock and perineum. It occurs more frequently in females than in males. Although the etiology of HS remains poorly understood, an inappropriate immunologic reaction to unknown antigen is suggested to play a role ${ }^{1}$. HS patients are at high risk for various metabolic diseases such as diabetes mellitus, dyslipidemia, and obesity ${ }^{2}$. Obesity is associated with the increased levels of cytokines such as tumor necrosis factor-alpha (TNF- $\alpha$ ), derived from adipocyte. And overexpressed TNF- $\alpha$ is shown to be related to the development and the severity of $\mathrm{HS}^{3}$.

Klinefelter syndrome (KS) is chromosomal abnormality in males, characterized by 47, XXY. Symptoms include gynecomastia, small testes and low testosterone levels. A number of cases have suggested that the coexistence of KS and autoimmune diseases may result from hypogonadism ${ }^{4}$ and decreased testosterone level ${ }^{5}$. Furthermore, patients with $\mathrm{KS}$ tend to have more metabolic diseases such as diabetes and obesity compared to normal male ${ }^{6}$. We report a rare case of HS who was already suffering KS, diabetes and metabolic syndrome. We thought these comorbidities played an important role in HS development and exacerbation in our case, although an exact mechanism is unknown.

\section{CASE REPORT}

A 30-year-old male patient was referred to the dermatologic clinic with purulent nodules and fistulas around the anus and perineal area. The patient was diagnosed with perianal abscesses. Then he received drainage and surgical resection several times for recurrent purulent nodules and took third generation cephalosporine antibiotics intermittently. But the symptoms got worse, so he was referred to Department of Dermatology, Soon Chun Hyang University College of Medicine, Cheonan. Multiple puru- 
lent nodules with tenderness and odor were present around the anus and perineum, accompanied by scar and sinus on the periphery (Fig. 1A). Hemorrhoid and anal fistula were ruled out by the clinical features and relapsing course of disease despite several times of surgical resection. Endoscopic examination revealed no significant findings for inflammatory bowel disease. Similar purulent nodules had previously existed on his both axilla which had disappeared after surgical resection. Only postoperative scar remained on axilla (Fig. 1B).

He has been diagnosed with KS (genetic testing, 47XXY) (Fig. 2), diabetes and cataracts in the past. Besides, he was obese (body mass index, $31.6 \mathrm{~kg} / \mathrm{m}^{2}$ ) and mentally retarded. His luteinizing hormone (LH) $(30.56 \mathrm{mIU} / \mathrm{ml})$ and follicle-stimulating hormone $(\mathrm{FSH})(52.40 \mathrm{mIU} / \mathrm{ml})$ levels were high and his testosterone level was low $(2.16 \mathrm{ng} / \mathrm{ml})$. We diagnosed him as HS (Hurley stage III) based on clinical findings, and then, we prescribed minocycline 400 mg daily for 15 months with intralesional triamcinolone injection twice a month. However, the patient experienced a recurrence of his symptoms repeatedly, so we did punch biopsy and tissue cultures from the perineal lesion to differentiate other infectious diseases. Histological examination revealed extensive fibrosis and inflammatory cell infiltration in the dermis to subcutis (Fig. 3). Tissue cultures were negative for bacteria, mycobacteria, and fungi. Finally, we started adalimumab (HUMIRA ${ }^{\circledR}$; AbbVie, Maidenhead, UK). He reported that symptoms including pain, pus, odor, and erythema improved immediately after the first treatment with adalimumab $160 \mathrm{mg}$. Currently, he is maintaining treatment with adalimumab $40 \mathrm{mg}$ weekly, and is showing continuous improvement (Fig. 4). We received the patient's consent form about publishing all photographic materials.

\section{DISCUSSION}

HS is a chronic inflammatory skin disease affecting apocrine gland-rich areas and presenting with painful nodules, abscesses, sinus tracts and scarring. The etiology of HS is still not clearly understood. But, its multi-factorial causes include folliculosebaceous anatomical abnormalities, genetic mutations, immunologic dysregulation and adnexal microbiota ${ }^{7}$.

Numerous studies have reported an increased level of TNF- $\alpha$ in the lesional and non-lesional skin of patients

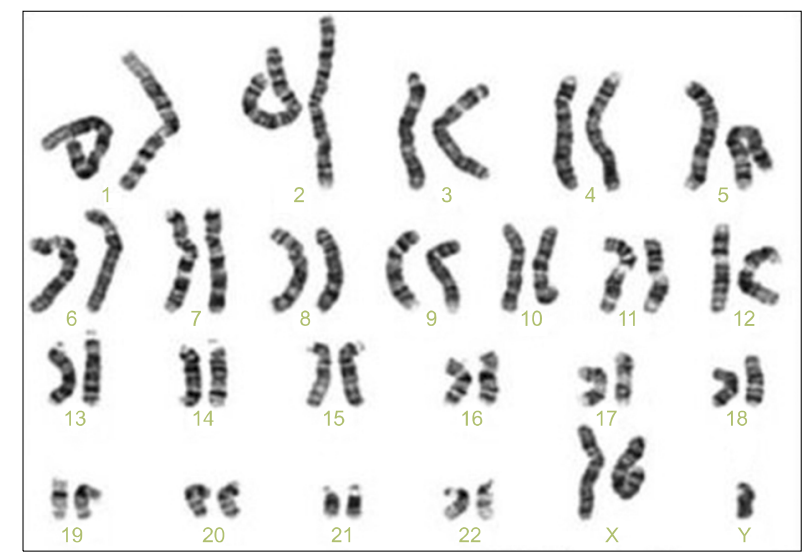

Fig. 2. Chromosome analysis of the patient demonstrating the karyotype of KS, 47XXY.
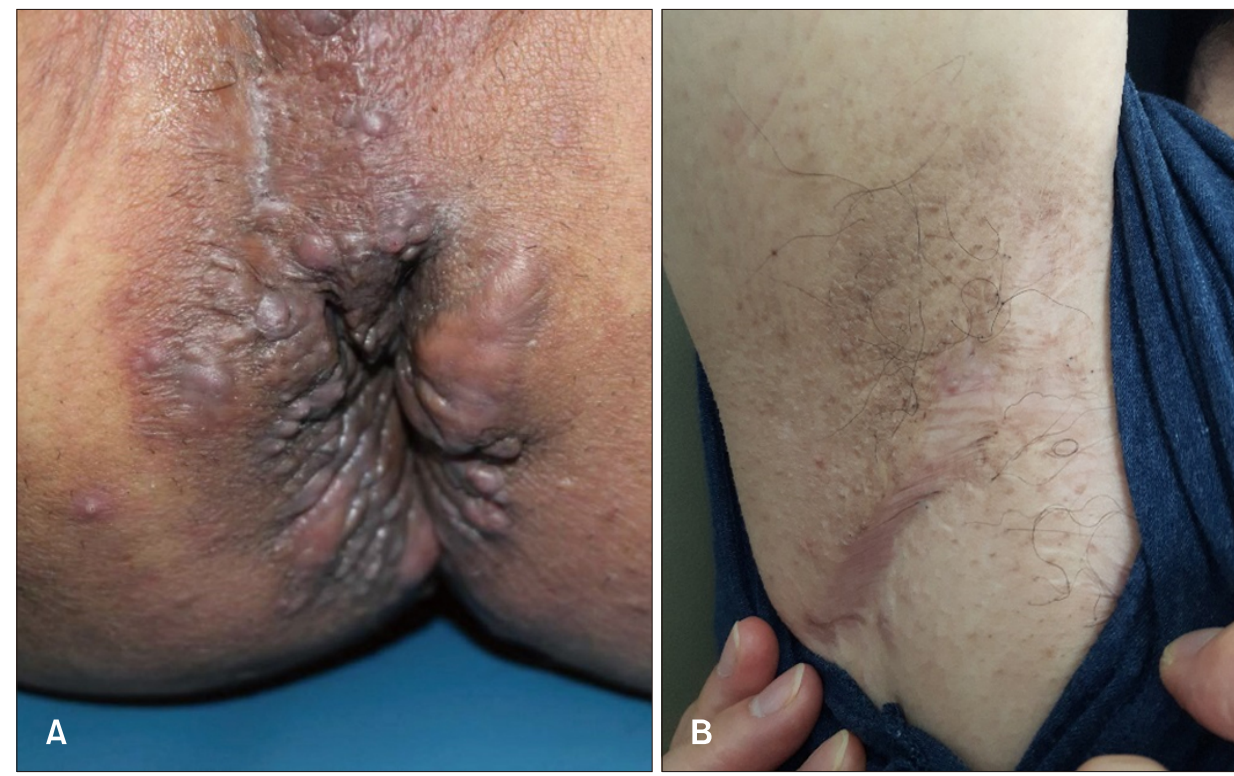

Fig. 1. (A) Deep-seated, inflamed and painful nodules with odorous discharge from perianal lesions and scar. (B) Postoperative scar on the axilla after surgical resection. 


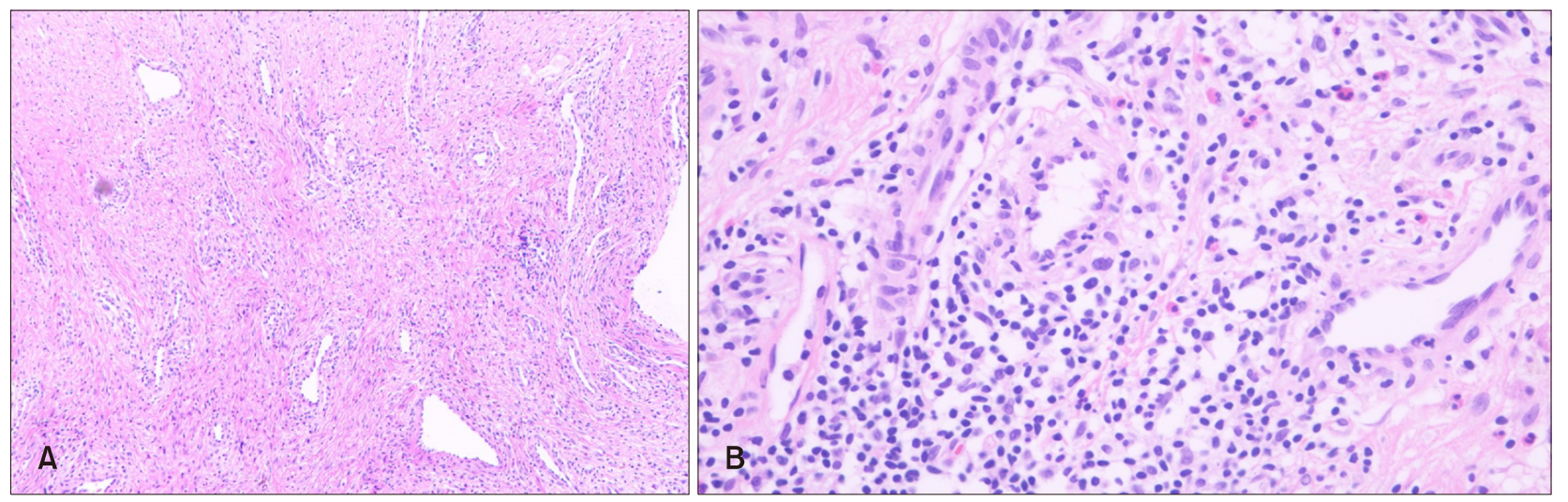

Fig. 3. (A, B) Histological examination revealed extensive fibrosis and inflammatory cell infiltration in the dermis to subcutis (H\&E: $A, \times 100 ; B, \times 400)$.

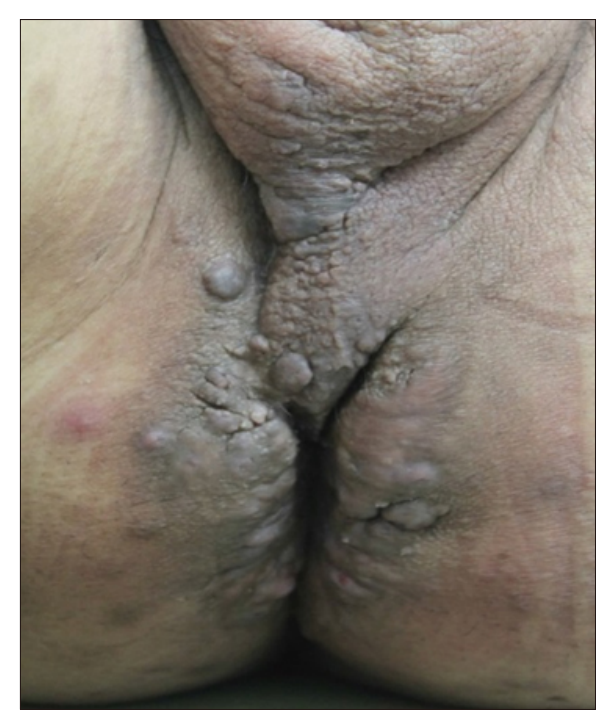

Fig. 4. Improved and decreased the painful and purulent nodules around the perianal lesions after 2nd adalimumab injection.

with $\mathrm{HS}$, becoming for the basis of targeted treatment using TNF- $\alpha$-biologics ${ }^{7}$. Previous studies have shown that when the patient is obese, macrophages in visceral fat secrete TNF- $\alpha$, which exacerbates $\mathrm{HS}^{8}$. Adalimumab $\left(\right.$ HUMIRA $^{\mathbb{R}}$ ) is the only approved TNF- $\alpha$ monoclonal antibody for the treatment of moderate to severe $\mathrm{HS}^{8}$. And its clinical effect was confirmed again in this case.

Although a consensus about the relationship between HS and $\mathrm{KS}$ is not established yet, low testosterone level in KS may have contributed to exacerbate our patient's condition by increasing TNF- $\alpha$. It may also be associated with the female predominance in $\mathrm{HS}^{8}$. And metabolic diseases such as diabetes and obesity may also synergistically affected the HS development in our case.

$\mathrm{KS}$, known as $47, \mathrm{XXY}$ or $\mathrm{XXXY}$, is the set of symptoms that result from two or more $X$ chromosomes in males due to nondisjunction of the sex chromosomes. Various studies have determined KS incidence to be 1:1,000 in live male birth and only $25 \%$ are diagnosed through life ${ }^{9}$. The prototype of KS is a tall stature, gynecomastia, small testes with azoospermia and hypergonadotrophic hypogonadism. Hormone levels of gonadotropins such as FSH and $\mathrm{LH}$ in KS patients are increased ${ }^{10}$ and testosterone levels are decreased to about half of the normal.

It has been reported that various $\mathrm{KS}$ comorbidities such as osteoporosis, metabolic disease and diabetes are related with hypogonadism ${ }^{9,11}$. Also, The coexistence of rheumatologic and autoimmune diseases associated with gonadal dysgenesis and/or KS is reported in many cases ${ }^{10}$. Seminog et al. $^{12}$ reported that the risk of female-predominant autoimmune disease, such as multiple sclerosis, acquired hypothyroidism, rheumatoid arthritis, Sjogren's syndrome and systemic lupus erythematosus, had increased in males with KS. Considering that KS is a disease related with an extra $\mathrm{X}$ chromosome (i.e., $\mathrm{XXY}$ ), it is suggested that the abnormality of $X$ chromosome contributed to the pathogenesis of the female-predominant autoimmunity in $\mathrm{KS}^{12}$. It is known that males with deficient sex hormones have more prevalence of autoimmune diseases, which means that testosterone may cause immunosuppression. Testosterone is suggested to have anti-inflammatory functions via both cellular and humoral immune suppression. Testosterone deficiency is linked with an increase of various inflammatory markers, such as TNF- $\alpha^{4}$. Furthermore, Bobjer et al. $^{13}$ found males with lower testosterone, but without manifestations of systemic disease, had higher levels of pro-inflammatory markers including TNF- $\alpha$ in young age. Many cases of coexistence of KS and autoimmune diseases with female predominance have been reported, but no case has been reported with $\mathrm{HS}^{12}$. As far as we know, this is the first case of a patient coexisting KS with HS. And 
adalimumab showed marked improvement in our case. Thus, we supposed this may be associated with an increased TNF- $\alpha$ in our patient. Herein, we report a rare case of HS in which KS was concurrently present and great response occurred to adalimumab therapy.

\section{ACKNOWLEDGMENT}

This work was supported by the Soon Chun Hyang University Research Fund.

\section{CONFLICTS OF INTEREST}

The authors have nothing to disclose.

\section{ORCID}

Ji Yeoun Shin, https://orcid.org/0000-0002-4859-4069

Jung Yeon Hong, https://orcid.org/0000-0001-7519-6042

Ho Jung Lee, https://orcid.org/0000-0001-9674-6429

Chang Yoon Sim, https://orcid.org/0000-0001-6622-8487

Young Lip Park, https://orcid.org/0000-0002-6532-3156

Jong Suk Lee, https://orcid.org/0000-0002-6554-7598

Sung Yul Lee, https://orcid.org/0000-0002-6995-4561

Jung Eun Kim, https://orcid.org/0000-0002-8399-8456

\section{REFERENCES}

1. Thorlacius L, Theut Riis $P$, Jemec GBE. Severe hidradenitis suppurativa responding to treatment with secukinumab: a case report. Br J Dermatol 2018;179:182-185.

2. Lim ZV, Oon HH. Management of hidradenitis suppurativa in patients with metabolic comorbidities. Ann Dermatol 2016;28:147-151.

3. Boer J. Does obesity cause a distinct phenotype of hidradenitis suppurativa? J Eur Acad Dermatol Venereol 2018; 32:e195-e196.

4. Baillargeon J, Al Snih S, Raji MA, Urban RJ, Sharma G, Sheffield-Moore $M$, et al. Hypogonadism and the risk of rheumatic autoimmune disease. Clin Rheumatol 2016;35: 2983-2987.

5. Oktenli C, Yesilova Z, Kocar IH, Musabak U, Ozata M, Inal A, et al. Study of autoimmunity in Klinefelter's syndrome and idiopathic hypogonadotropic hypogonadism. J Clin Immunol 2002;22:137-143.

6. Jiang-Feng M, Hong-Li X, Xue-Yan W, Min N, Shuang-Yu L, Hong-Ding $X$, et al. Prevalence and risk factors of diabetes in patients with Klinefelter syndrome: a longitudinal observational study. Fertil Steril 2012;98:1331-1335.

7. Maarouf M, Clark AK, Lee DE, Shi VY. Targeted treatments for hidradenitis suppurativa: a review of the current literature and ongoing clinical trials. J Dermatolog Treat 2018; 29:441-449.

8. Napolitano M, Megna M, Timoshchuk EA, Patruno C, Balato N, Fabbrocini G, et al. Hidradenitis suppurativa: from pathogenesis to diagnosis and treatment. Clin Cosmet Investig Dermatol 2017;10:105-115.

9. Høst C, Skakkebæk A, Groth KA, Bojesen A. The role of hypogonadism in Klinefelter syndrome. Asian J Androl 2014; 16:185-191.

10. Kobak S, Yalçin M, Karadeniz M, Oncel G. Coexistence of ankylosing spondylitis and Klinefelter's syndrome. Case Rep Rheumatol 2013;2013:543953.

11. Belling K, Russo F, Jensen AB, Dalgaard MD, Westergaard $D$, Rajpert-De Meyts E, et al. Klinefelter syndrome comorbidities linked to increased $X$ chromosome gene dosage and altered protein interactome activity. Hum Mol Genet 2017; 26:1219-1229.

12. Seminog $O O$, Seminog AB, Yeates D, Goldacre MJ. Associations between Klinefelter's syndrome and autoimmune diseases: English national record linkage studies. Autoimmunity 2015;48:125-128.

13. Bobjer J, Katrinaki M, Tsatsanis C, Lundberg Giwercman $Y$, Giwercman A. Negative association between testosterone concentration and inflammatory markers in young men: a nested cross-sectional study. PLoS One 2013;8:e61466. 